\title{
Metal Stent Placement in the Afferent Loop Obstructed by Peritoneal Metastases-Experience of Five Cases
}

\author{
Yoshihide Kanno, Tetsuya Ohira, Yoshihiro Harada, Yoshiki Koike, Taku Yamagata, Megumi Tanaka, Tomohiro Shimada and Kei Ito \\ Department of Gastroenterology, Sendai City Medical Center, Sendai, Japan
}

Afferent loop syndrome is often difficult to resolve. Among patients with afferent loop syndrome whose data were extracted from databases, 5 patients in whom metal stent placement was attempted were included and evaluated in this study. The procedure was technically successful without any adverse events in all patients. Metal stent(s) was placed with an endoscope in the through-the-scope manner in 4 patients and via a percutaneous route in 1 patient. Obvious clinical efficacy was observed in all patients. Adverse events related to the procedure and stent occlusion during the follow-up period were not observed. Metal stent placement for malignant obstruction of the afferent loop was found to be safe and feasible. Clin Endosc 2018;51:299-303

Key Words: Afferent loop syndrome; Self expandable metallic stents; Balloon enteroscopy; Intestinal obstruction; Palliative care

\section{INTRODUCTION}

Afferent loop syndrome induced by the obstruction of a surgically reconstructed afferent loop is relatively rare but often difficult to resolve. The obstruction can be conservatively treated without any physical intervention when it is reversible; however, if it is irreversible, it must be treated by surgical intervention including adhesiolysis, intestinal bypass, or partial resection of the intestine. For patients in whom this syndrome is due to malignant obstruction, such surgical treatments are extremely challenging if the malignancy is in the most advanced stage.

Self-expanding metal stents for endoscopic placement into the gastrointestinal tract have been developed and are commercially available for the treatment of obstructions in the

Received: December 5, 2017 Revised: January 5, 2018

Accepted: January 23, 2018

Correspondence: Yoshihide Kanno

Department of Gastroenterology, Sendai City Medical Center, 5-22-1, Miyagino-ku, Sendai 983-0824, Japan

Tel: +81-22-252-1111, Fax: +81-22-252-9431, E-mail: yoshi-hk@openhp.or.jp ORCID: https://orcid.org/0000-0002-2044-5483

(cc) This is an Open Access article distributed under the terms of the Creative Commons Attribution Non-Commercial License (http://creativecommons.org/ licenses/by-nc/3.0) which permits unrestricted non-commercial use, distribution, and reproduction in any medium, provided the original work is properly cited. esophagus, stomach, duodenum, colon, and rectum. However, metal stents dedicated for use in the small intestine have not been developed because the demand is extremely low, and stents for the colon and rectum from several major manufacturers have almost the same specifications as those for the stomach and duodenum. While the placement of such stents into the small intestine has been reported to be efficacious and safe, ${ }^{1-3}$ the number of published reports is lacking and include a relatively small number of cases. Therefore, the safety and efficacy of this treatment approach has not been sufficiently validated.

In addition, there have been few reports on the placement of metal stents into the afferent loop. If such an intervention were shown to be feasible and effective, the treatment strategy for afferent loop syndrome would evolve.

At our institution, we have treated patients with malignant afferent loop obstruction using metal stent placement, and we report the results herein.

\section{CASE REPORT}

\section{Methods}

To identify patients with afferent loop syndrome for this 
study, the registration system for national insurance reimbursement claims, discharge summaries, and the prospectively maintained database of the placement of gastrointestinal stents at Sendai City Medical Center between 2009 and 2016 were searched. All patients in whom metal stent placement had been attempted or performed were included in this study.

Metal stents for endoscopic through-the-scope insertion into the colon, which were commercially available at the time (Enteral WallFlex; Boston Scientific Japan K.K., Tokyo, Japan or Colorectal Niti-S; Century Medical, Tokyo, Japan), were used. The patients included in the search were not only those in whom a stent was deployed with an endoscope, but also those in whom it was deployed via a percutaneous route or a route created by an endoscopic ultrasound-guided puncture. While a stent $22 \mathrm{~mm}$ in diameter (WallFlex or Niti-S) was deployed when a scope with a sufficiently wide working channel could be advanced into the obstructed intestine, a stent $18 \mathrm{~mm}$ in diameter (Niti-S) was placed when the utilized scope had a thinner channel, e.g., traditional colonoscopes or balloon enteroscopes.

The technical success of metal stent placement, adverse events related to the procedure, clinical efficacy, and prognosis after the procedure were retrospectively evaluated. Technical success was defined as successful placement of a metal stent into the target intestine with resolution of the obstruction. Adverse events were defined as any unfavorable symptoms such as abdominal pain, bleeding, perforation, or any clinically important events that required additional treatment or interventions. Clinical effectiveness was defined as follows: Good, complete disappearance of acute infectious disease (cholangitis and enteritis) and symptoms derived from intestinal obstruction; Fair, improvement of such a condition; Poor, little or no efficacy in the improvement of such a condition.

Written informed consent was obtained prior to the procedure in all patients. This study was approved by the institutional review board of our center.

\section{Results}

The data of 10 patients registered as having "afferent loop syndrome" were extracted from the electronic database for the national insurance reimbursement claim. Among them, metal stent placement in the afferent loop was attempted or performed in 3 patients. Among the other 5 patients, 1 recovered with conservative treatment, 3 were treated with surgical adhesiolysis, and 1 underwent bypass surgery. Two other patients in whom treatment of this syndrome had been attempted by metal stent placement were detected in the database of the discharge summaries and in the database of gastrointestinal stents. Thus, a total of 5 patients were evaluated in this study.

A summary of the 5 patients is shown in Table 1. Three pa- tients complained of abdominal pain and pyrexia (\#2, \#3, and \#4 in Table 1), whereas the other 2 patients had no symptoms. Blood biochemistry findings revealed elevated levels of C-reactive protein and biliary enzymes, indicating varying levels of infectious enteritis and acute cholangitis in all patients. Computed tomography showed the obstructed intestine with dilation of the upstream afferent loop and bile duct in all patients.

Metal stent(s) placement was technically successful in all patients. While 4 patients underwent stent placement with an endoscope (Fig. 1), 1 underwent placement via a percutaneous route created between the skin and duodenum via the liver in a previously performed emergency intervention (Fig. 2). The scope utilized in patient \#2 was a prototype of a short single-balloon enteroscope such as SIF-H290S (Olympus, Tokyo, Japan), with a $152-\mathrm{cm}$ effective length and a $3.2-\mathrm{mm}$ wide working channel. The scopes utilized in the other 3 patients were commercially available from Olympus. In patient \#1, another stricture on the liver side of the afferent loop was revealed after 1 stent was deployed at the obstructed site, requiring the deployment of 1 more stent in the same session. In patient \#5, a metal stent (Niti-S, $18 \mathrm{~mm}$ wide, $12 \mathrm{~cm}$ long) could not be deployed using a short double balloon enteroscope (EI580BT; Fujifilm Medical, Tokyo, Japan) because the delivery catheter could not be inserted beyond the stricture despite successful insertion of a guidewire. Two days later, the procedure was attempted with a short single balloon enteroscope (SIF-H290S; Olympus) and was successfully completed.

All stents deployed at the stricture were clinically effective (good) and allowed hospital discharge.

No adverse events related to the procedure were observed. Patient \#2 suffered from liver abscess 10 months after the procedure, although obstruction of the afferent loop did not recur. Additional interventions such as percutaneous or endoscopic drainage were not performed as they were considered to entail excessive risk owing to the patient's debilitated condition. $\mathrm{He}$ finally succumbed 12 months after the procedure due to the advance of the malignancy and uncontrollable infection.

\section{DISCUSSION}

This study showed a high success rate of metal stent placement in the afferent loop without any adverse events. However, the actual success rate is possibly lower because there might have been patients who underwent unsuccessful procedures who were not registered as having afferent loop syndrome in spite of having been diagnosed with the syndrome. However, all patients in whom metal stent placement was attempted after successful insertion of an endoscope into the obstruction were 


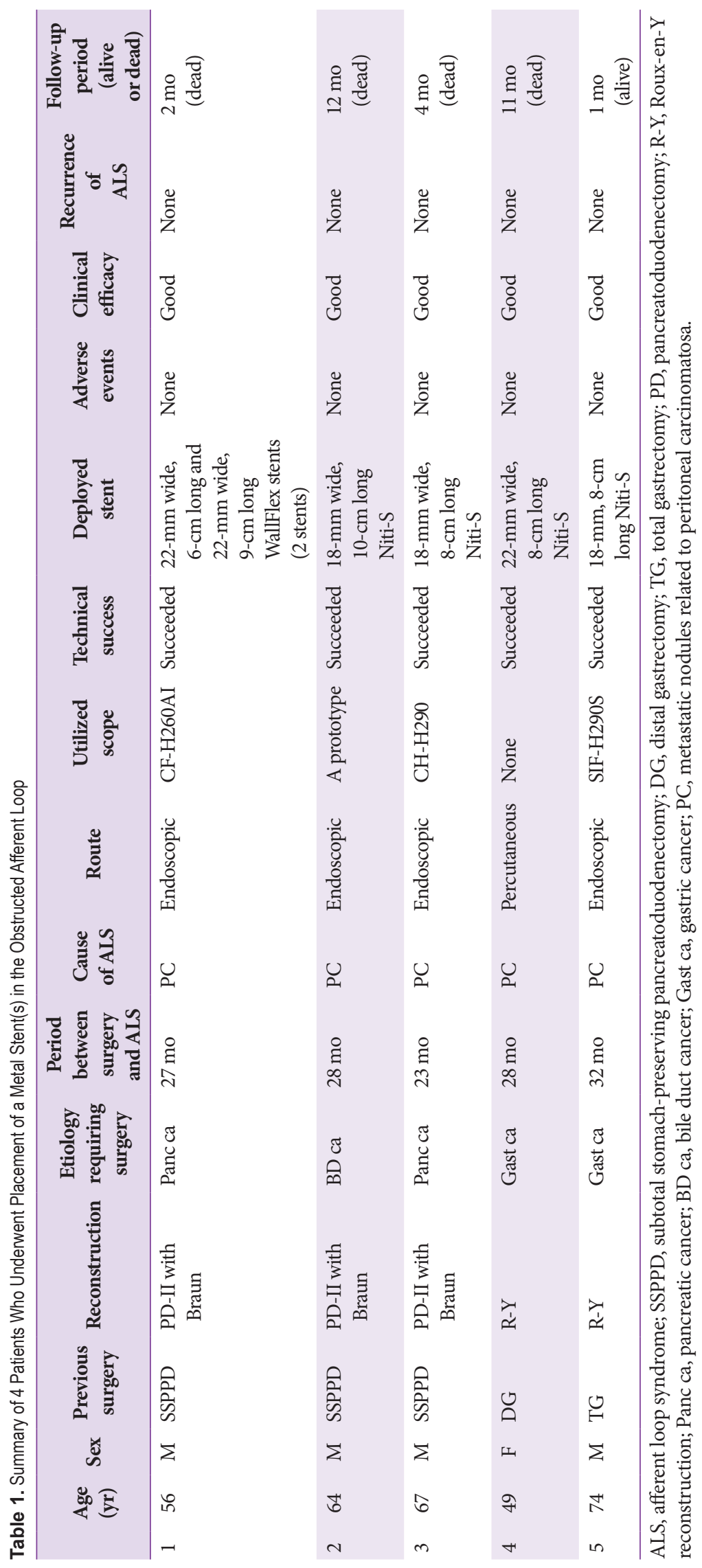



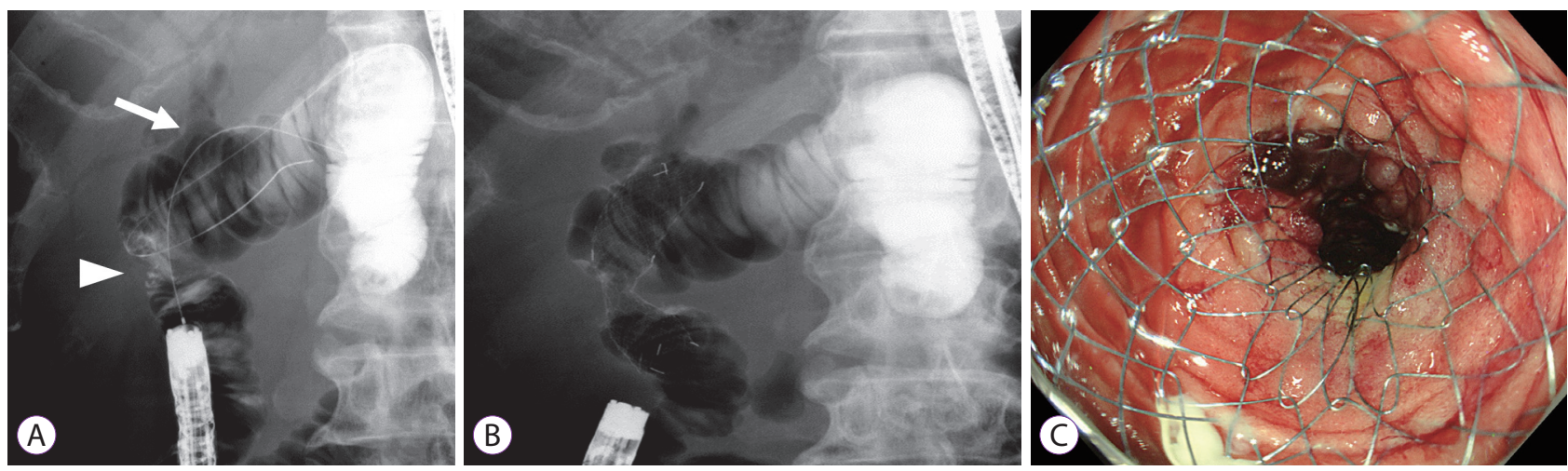

Fig. 1. Endoscopic placement of a metal stent in the obstructed afferent loop (patient \#3). Arrowhead, obstruction; arrow, bilio-enteric anastomosis.
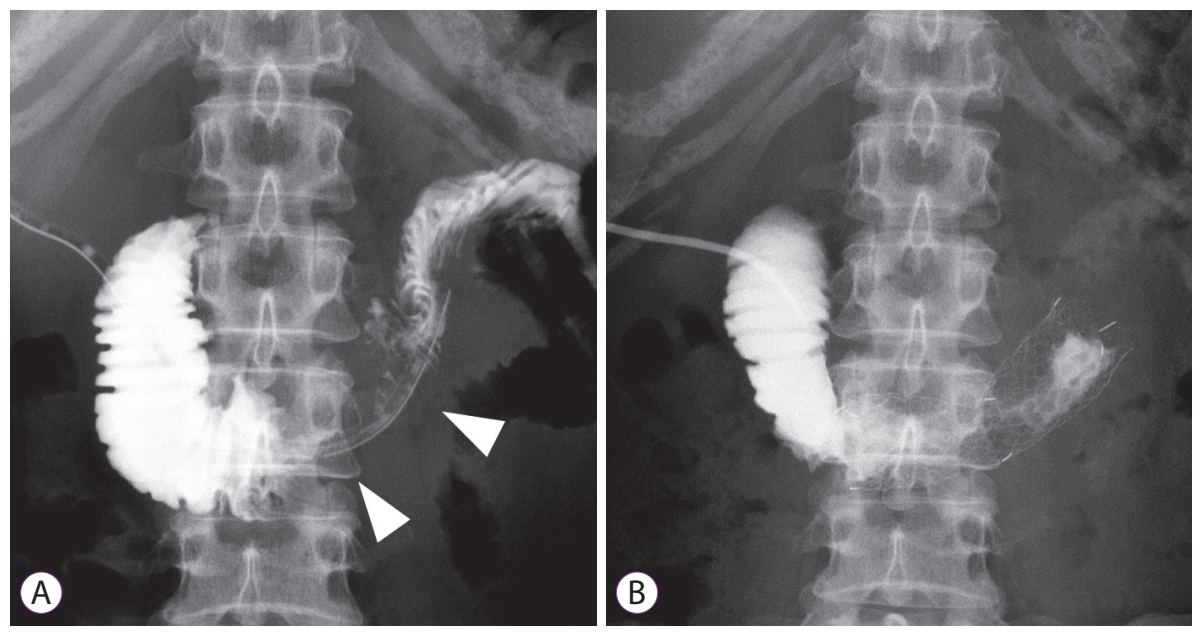

Fig. 2. Metal stent placement in the obstructed afferent loop via the previously created percutaneous route (patient \#4). Arrowhead, obstruction.

registered in the database of endoscopic metal stent placement at our center; therefore, the success rate of metal stent placement after successful insertion of a scope is sufficiently high. Some reports have shown similarly favorable outcomes of endoscopic through-the-scope placement of metal stents in the afferent loop in a small population. ${ }^{1-3}$ Other studies have also reported the technical feasibility of deployment through an overtube utilized for insertion of a balloon enteroscope. ${ }^{4-7}$ However, these studies had the same limitations due to their retrospective design. Since the number of patients in whom endoscopic treatment was abandoned is unknown, the true success rate would likely be lower.

The safety of such deployment was also found to be high. Enteral metal stents that were developed for other intestinal tracts could be applied for the small intestine including the afferent loop. Moreover, some studies have reported safe placement of a second stent using the stent-in-stent technique in the small intestine. ${ }^{8,9}$ Therefore, further studies on metal stent placement in the small intestine with a prospective design and a large sample size in multiple institutions are warranted.

In cases with severe infection derived from obstruction of the afferent loop, emergency drainage is required. Endoscopic treatment such as placement of a stent or a drainage tube is sometimes inappropriate in an emergency context because it can involve a prolonged procedure time with increased risk of cardiorespiratory events under sedation and scope insertion. Moreover, the scopes are not always successfully advanced into the obstructed intestine. In such a situation, percutaneous drainage is sufficiently acceptable. In cases with severe cholangitis, the bile duct can be directly punctured. After recovery from the infectious state, the punctured tract can be utilized for metal stent deployment into the obstructed intestine. The successful performance of such a technique precludes endoscope insertion with its associated risks of adverse, procedure-related events and technical failure.

The critical limitation of the present study is its extremely small study population. However, given that neither the feasibility nor the safety of the technique has been established, increased numbers of patients for whom traditional treatment is inappropriate must be accumulated, at which point the feasibility and safety can be accurately evaluated.

In conclusion, metal stent placement for malignant obstruc- 
tion of the afferent loop was found to be safe and feasible. Further studies with a large population in a multicenter setting are necessary to validate our findings.

\section{Conflicts of Interest}

The authors have no financial conflicts of interest.

\section{REFERENCES}

1. Pannala R, Brandabur JJ, Gan SI, et al. Afferent limb syndrome and delayed GI problems after pancreaticoduodenectomy for pancreatic cancer: single-center, 14-year experience. Gastrointest Endosc 2011;74:295302.

2. Huang J, Hao S, Yang F, et al. Endoscopic metal enteral stent placement for malignant afferent loop syndrome after pancreaticoduodenectomy. Wideochir Inne Tech Maloinwazyjne 2015;10:257-265.

3. Kim JK, Park CH, Huh JH, et al. Endoscopic management of afferent loop syndrome after a pylorus preserving pancreatoduodenecotomy presenting with obstructive jaundice and ascending cholangitis. Clin
Endosc 2011;44:59-64.

4. Ross AS, Semrad C, Waxman I, Dye C. Enteral stent placement by double balloon enteroscopy for palliation of malignant small bowel obstruction. Gastrointest Endosc 2006;64:835-837.

5. Hayashi Y, Yamamoto H, Kita H, et al. Education and imaging. Gastrointestinal: metallic stent for an obstructing jejunal cancer. J Gastroenterol Hepatol 2006;21:1861.

6. Kida A, Matsuda K, Noda Y. Endoscopic metallic stenting by double-balloon enteroscopy and its overtube for malignant gastrointestinal obstruction as palliative treatment. Dig Endosc 2013;25:552-553.

7. Nakahara K, Okuse C, Matsumoto N, et al. Enteral metallic stenting by balloon enteroscopy for obstruction of surgically reconstructed intestine. World J Gastroenterol 2015;21:7589-7593.

8. Popa D, Ramesh J, Peter S, Wilcox CM, Mönkemüller K. Small bowel stent-in-stent placement for malignant small bowel obstruction using a balloon-assisted overtube technique. Clin Endosc 2014;47:108-111.

9. Chok AY, Koh YX, Lee J, Wong AS. Secondary stent-in-stent self-expanding metallic stent placement for early stent occlusion due to mucosal oedema in the gastrojejunal anastomosis after palliative subtotal gastrectomy for locally advanced gastric cancer. Singapore Med J 2013;54:e240-e243. 